\title{
Proceedings of the 11th Brazilian Workshop on Semiconductor Physics
}

This special volume of the Brazilian Journal of Physics contains the proceedings of the 11th Brazilian Workshop on Semiconductor Physics (BWSP-11), held in Fortaleza, Ceará, from 9 to 14 March, 2003. Over one hundred participants came to this event, including 12 invited speakers. There were 82 posters and 13 selected oral presentations, in parallel with the invited talks. The articles that are now published, revised and refereed according to the usual standards of the Braz. J. Phys., provide a fair overview of the program of the meeting, covering a number of modern and relevant topics in semiconductor physics.

The first Brazilian Workshop on Semiconductor Physics was held in 1983, in Campinas, São Paulo. It has been relevant for promoting and consolidating this area, as well as for gathering different research groups in Brazil. As a mix of a Summer school and regular conference, it has been very important for young researchers. It was the first time that this meeting was held in the Northeast of Brazil, which indicates the growth and maturity of the scientific community in this region. The program of BWSP-11 is very close to the program of the last meeting, covering all of the aspects of semiconductor physics, and was closely tuned to the programs of other important conferences in this area.

The members of the National, Program, and Local Committees, and the sponsors of the 10th Brazilian Workshop on Semiconductor Physics, are listed in the following page. We thank the invited and contributing authors for preparing their manuscripts, and our colleagues in the Program Committee for their active involvement in the refereeing process.

G. A. Farias

Guest Editor 